\title{
Patterns of geographic variation of thermal adapted candidate genes in Drosophila subobscura sex chromosome arrangements
}

Pedro Simões ${ }^{1,2^{*}}$ (1) and Marta Pascual ${ }^{1}$

\begin{abstract}
Background: The role of chromosomal arrangements in adaptation is supported by the repeatable clinal variation in inversion frequencies across continents in colonizing species such as Drosophila subobscura. However, there is a lack of knowledge on the genetic variation in genes within inversions, possibly targets of climatic selection, across a geographic latitudinal gradient. In the present study we analysed four candidate loci for thermal adaptation, located close to the breakpoints, in two chromosomal arrangements of the sex (A) chromosome of Drosophila subobscura with different thermal preferences. Individual chromosomes with $\mathrm{A}_{2}$ (the inverted arrangement considered warm adapted) or $A_{S T}$ (the standard ancestral arrangement considered cold adapted) were sequenced across four European localities at varying latitudes, up to $\sim 2500 \mathrm{Kms}$ apart.

Results: Importantly, we found very low differentiation for each specific arrangement across populations as well as no clinal patterns of genomic variation. This suggests wide gene exchange along the cline. Differentiation between the sex chromosome arrangements was significant in the two more proximal regions relative to the AsT orientation but not in the distal ones, independently of their location inside or outside the inversion. This can be possibly due to variation in the levels of gene flux and/or selection acting in these regions.

Conclusions: Gene flow appears to have homogenized the genetic content within-arrangement at a wide geographical scale, despite the expected diverse selective pressures in the specific natural environments of the different populations sampled. It is thus likely that the inversion frequency clines in this species are being maintained by local adaptation in face of gene flow. The differences between arrangements at non-coding regions might be associated with the previously observed differential gene expression in different thermal regimes. Higher resolution genomic scans for individual chromosomal arrangements performed over a large environmental gradient are needed to find the targets of selection and further elucidate the adaptive mechanisms maintaining chromosomal inversion polymorphisms.
\end{abstract}

Keywords: Chromosomal arrangements, Drosophila subobscura, UTR variation, Genetic differentiation, Gene flow, Geographic variation, Clinal variation, Climatic selection, Thermal adaptation

\section{Background}

There is increasing evidence of the role of chromosomal inversion polymorphisms in adaptation and speciation $[1,2]$. Several lines of evidence attest this namely: (1) the repeated occurrence of latitudinal clinal variation across

\footnotetext{
* Correspondence: pmsimoes@fc.ul.pt

'Departament de Genètica, Microbiologia i Estadística and IRBio, Facultat de Biologia, Universitat de Barcelona, 08028 Barcelona, Spain

${ }^{2}$ Present Address: cE3c - Centre for Ecology, Evolution and Environmental

Changes, Faculdade de Ciências, Universidade de Lisboa, Campo Grande, 1749-016 Lisbon, Portugal
}

(c) The Author(s). 2018 Open Access This article is distributed under the terms of the Creative Commons Attribution 4.0 International License (http://creativecommons.org/licenses/by/4.0/), which permits unrestricted use, distribution, and reproduction in any medium, provided you give appropriate credit to the original author(s) and the source, provide a link to the Creative Commons license, and indicate if changes were made. The Creative Commons Public Domain Dedication waiver (http://creativecommons.org/publicdomain/zero/1.0/) applies to the data made available in this article, unless otherwise stated.

multiple invaded continents, particularly in Drosophila species ([3-6]; (2) consistent associations between inversions and several relevant phenotypic traits [7-13]; and (3) inversion frequency changes beyond genetic drift expectations across replicated populations adapting to imposed laboratory conditions [14-16].

Several hypotheses have been advanced to explain the adaptive character of inversions (see [1]), with the most widely accepted hypotheses relying on recombination reduction in inversion heterokaryotypes and consequent protection of genes with positive effects on fitness $[17,18]$. 
The coadaptation hypothesis [17] postulates that specific favourable combinations of alleles interacting epistatically within a particular inversion arise as an adaptive response to a given environment. The local adaptation hypothesis [18] states that the spread of a new inversion results from advantageous locally adapted alleles captured within the inverted segment, without epistasis being necessarily involved. Inversion frequencies changes will result from a balance between selection and migration. A general expectation in both these scenarios is that there is genetic differentiation between inversion types, as a result of local adaptation shaping the genetic content of different inversions. Coalescent models suggest that this differentiation is likely higher near breakpoints - where recombination is greatly reduced - and near locally adapted alleles within the inversion [19]. In addition, under the coadaptation hypothesis there is a clear expectation of genetic differentiation within inversions sampled from different geographical populations, as a result of population-specific epistatic interactions evolving in response to different environmental conditions [20]. However, this pattern might not be exclusive of the co-adaptation model as some within-inversion differentiation might occur depending on the balance between migration and selection on locally adapted alleles (with or without epistasis involved) as postulated by the local adaptation.

Empirical studies on the molecular variation associated with inversions have frequently detected high levels of genetic differentiation between chromosomal arrangements ([21-27] but see [28]). This pattern is consistent with the aforementioned hypotheses and is expected as a result of reduced recombination in inversion heterokaryotypes leading to distinct genetic pools. Studies in the O chromosome of $D$. subobscura found an extensive genetic differentiation between arrangements occurring regardless of the distance to the nearest breakpoint, with selection likely implicated [29, 30]. On the other hand, several studies have reported a general lack of measurable genetic differentiation within arrangements sampled from different populations ([20,31-33], contrary to the expectations of the coadaptation hypothesis. An important exception is the genetic clinal variation within the inversion In(3R)Payne of Drosophila melanogaster, both in North American and Australian localities, implicating a role of this inversion in the response to climatic selection $[6,31,34]$. In addition, there is some evidence for epistasis - as expected under the coadaptation model based on patterns of linkage disequilibrium (LD) with regions within arrangements showing high levels of LD and differentiation interspersed with other regions of low LD [20,31] as well as LD between arrangements and genes located outside them [30].
One of the most emblematic examples of the adaptive role of chromosomal polymorphisms comes from Drosophila subobscura, a species presenting repeatable clinal patterns for inversion frequencies across Europe, the ancestral area, and North and South America [3]. Thermal adaptation or associated factors might be implicated in such patterns. Evidence for this hypothesis comes from the association between temporal frequency changes of D. subobscura chromosomal arrangements and increased temperature due to climate warming at a worldwide scale [5]. Also, seasonal inversion frequency changes in D. subobscura have been reported, even shortly responding to heat waves [35] as well as associations between inversions and several phenotypic traits, including thermal preference and tolerance [36-38] and body size [10, 39]. Importantly, differences in gene expression patterns were also observed in D. subobscura from a colonizing South American population evolving in the laboratory at different thermal regimes $\left(13,18\right.$ and $\left.22{ }^{\circ} \mathrm{C}\right)$; with an enrichment for candidate genes located inside arrangements [40]. This suggests that different inversions may contribute to the maintenance of genes with differential expression and potential impact on fitness. Indeed, this was the case in $D$. pseudoobscura with multiple genes presenting expression differences among arrangements [41]. Consequently, it is relevant to analyse overall patterns of molecular variation in these candidate genes in the vicinity/within the $D$. subobscura inversions as well as address their variation across the species geographic gradient to search for targets of climatic selection.

Pegueroles et al. [33] analysed 6 of these genes previously associated with thermal adaptation located in the O chromosome of D. subobscura and found no differentiation for the same arrangement between Barcelona and Greece indicating high levels of gene flow and the absence of geographic varying selection at least in those two southern regions. In a study in D. subobscura involving several localities across western Europe, Simões et al. [32] using microsatellite loci also detected low within-arrangement differentiation in several inversions of two autosomes ( $J$ and $U$ ) and the sex chromosome (A) confirming an extensive gene flow in this species (see also [42, 43]). Nevertheless, significant clinal patterns at few specific microsatellite loci within arrangements emerged (one in the distal region of $A_{2}$ ), suggesting adaptation at neighbouring loci.

The aim of the present study is to examine the genetic variation associated with two arrangements of the sexual chromosome of Drosophila subobscura across a geographic gradient. Specifically, we intend to (1) characterize genetic variation and differentiation between arrangements, addressing the impact of inverted regions at the genetic level; (2) analyse patterns of within-arrangement differentiation, searching for evidence of different epistatic 
combinations in different populations; (3) identify latitudinal patterns of genetic variation, which might indicate targets of climatic selection at a geographical scale. In this study we targeted $\mathrm{A}_{\mathrm{ST}}$ and $\mathrm{A}_{2}$, the two most frequent inversions of the A chromosome in our sampled localities. These two arrangements present clinal variation of frequencies in Europe, as well as in the two recently colonized American hemispheres, with $\mathrm{A}_{2}$ increasing frequency towards warmer locations and $\mathrm{A}_{\mathrm{ST}}$ showing the corresponding opposite pattern [32, 44]. We analysed DNA sequence variation in four gene regions (PhKgamma, $U b c-E 2 H, H f w$ and Nipsnap). These genes are good candidates to search for evidences of climatic latitudinal selection associated with the Drosophila subobscura inversion clines as they (1) have been previously implicated in thermal adaptation ([40], see also above) and (2) are located relatively close to inversion breakpoints, with reduced recombination in these regions possibly protecting genetic variants and showing the footprint of selection.

\section{Methods}

Fly samples and chromosomal inversion scoring

Wild Drosophila subobscura samples were collected from four European locations (Fig. 1). Samples from Málaga (Mal, $36^{\circ} 43^{\prime} \mathrm{N}$, Spain) and Rasquera (Ras, $40^{\circ}$ 57 'N, Spain) were obtained in October 2008 with a total of 169 and 152 individuals, respectively. Samples from Dijon (Dij, $47^{\circ} 18^{\prime} \mathrm{N}$, France) and Groningen (Gro, $57^{\circ} 13^{\prime} \mathrm{N}$,
Netherlands) were obtained in August/September 2009 with a total of 344 and 326 individuals, respectively [45]). These locations vary greatly in environmental conditions, for instance the two most extreme sampled locations have presented clearly contrasting range of yearly average temperatures $\left(7-31{ }^{\circ} \mathrm{C}\right.$ in Málaga vs. $0-23{ }^{\circ} \mathrm{C}$ in Groningen) and also average precipitation values (44 $\mathrm{mm}$ in Málaga vs. $75 \mathrm{~mm}$ in Groningen) between 2000 and 2012. We performed the collections in the late summer/early fall to capture the second peak of abundance for the species in the different locations allowing a more reliable comparisons between warmer and colder populations [46].

Our study complies with National and International guidelines, either involving field collections or experimentation in animals. We use Drosophila subobscura, which is not a threatened species nor requires legal ethical consent for experimentation.

This study focused on the two most frequent arrangements present on the acrocentric A (sexual) chromosome $\left(\mathrm{A}_{\mathrm{ST}} / \mathrm{A}_{2}\right)$, and the only two arrangements present in all analysed locations (Fig. 1). $\mathrm{A}_{\mathrm{ST}}$ is considered an ancestral chromosome arrangement and $\mathrm{A}_{2}$ a derived medium-sized inversion $(\sim 7.1 \mathrm{Mb})$ with an estimated age of 160.000 years [23]. The breakpoints of $\mathrm{A}_{2}$ (Fig. 2) are $8 \mathrm{C} / \mathrm{D}-12 \mathrm{C} / \mathrm{D}$ [47]. In order to score the chromosomal arrangements of the A chromosome, wild-caught males or $F_{1}$ male descendants from wild isofemale lines were individually crossed with virgin females of the $c h c u$

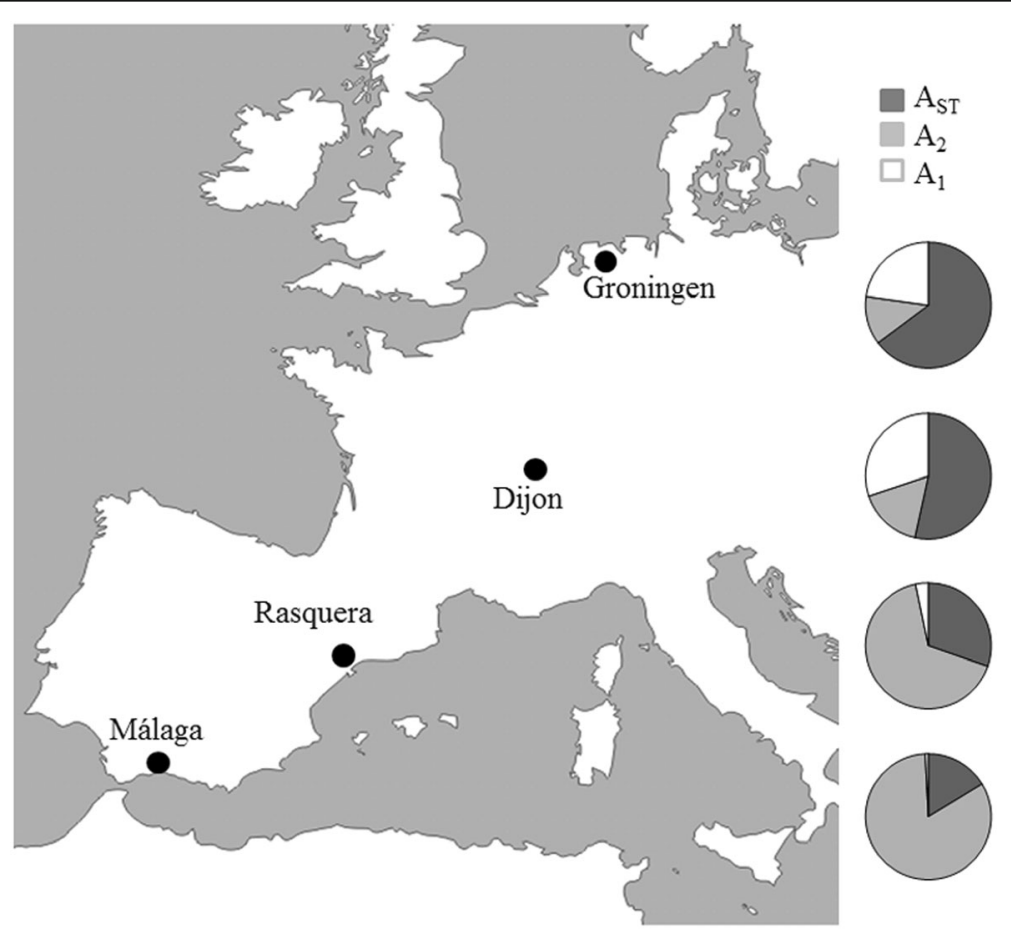

Fig. 1 Sampling sites of D. subobscura and frequency, as coloured pies for each locality, of the three sex chromosome arrangements detected (data from [25]). $A_{S T}$ - dark grey, $A_{2}$ - light grey; $A_{1}$ - white 


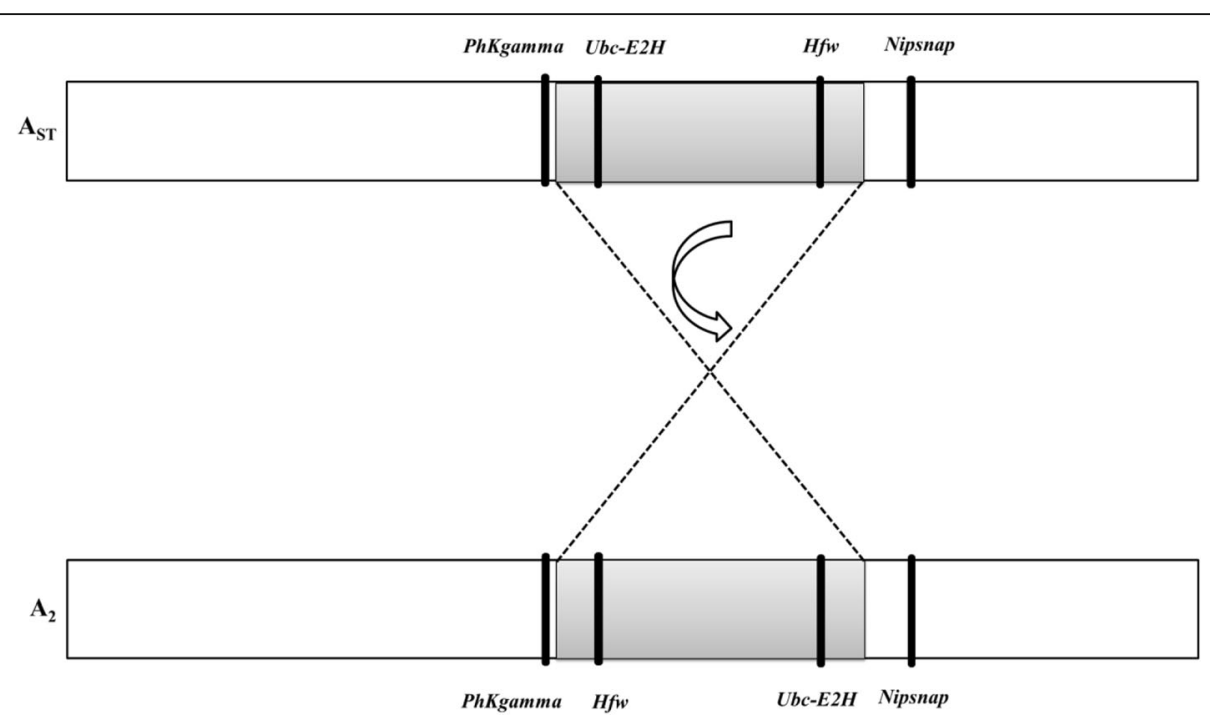

Fig. 2 Cytological location of the genes analysed. The grey box represents the region inverted in the $A_{2}$ arrangement. Black bars indicate the cytological location of each gene in each chromosomal arrangement

strain, homokaryotypic for the $\mathrm{A}_{\mathrm{ST}}$ arrangement. Polytene chromosomes from salivary glands of one female larva descendant per cross were stained with $2 \%$ orcein in $60 \%$ acetic/lactic acid (50:50) (see details in [32]). This protocol allowed identifying the chromosomal arrangement of each parental male from the chromosomal configuration of their female larva progeny.

\section{Genomic regions, DNA extraction and sequencing}

DNA was individually extracted from wild males (or male descendants of wild females) whose chromosomal arrangements $\left(\mathrm{A}_{2}\right.$ or $\left.\mathrm{A}_{\mathrm{ST}}\right)$ had been previously scored as described above. By amplifying and directly sequencing males we avoided the problems associated with sequencing of diploid data. The karyotype of these males was inferred from their descendants (see above) making it possible to obtain both genetic and karyotypic data for the same individual. Genomic DNA extraction was performed following the protocol described in [48]. The four genomic regions analysed were localized again by in situ hybridization with digoxigenin following standard protocols [49], as some genes in Laayouni et al. [40] have proved to be mislocated [33]. All probes hybridized in the same band previously reported [40] with the exception of Nipsnap that was localized nearby but outside the inversion (see Fig. 2 and Additional file 1). Thus, PhKgamma (cytological location 8C; CG1830) and Nipsnap (13B; CG9212) are located outside the $\mathrm{A}_{2}$ inversion, and $U b c-E 2 H$ (9A; CG2257) and Hfw (12A; CG3095) within that inversion (Fig. 2). Following the $\mathrm{A}_{\mathrm{ST}}$ arrangement, being the ancestral one, we will refer to PhKgamma and $U b c-E 2 H$ as the proximal loci, the region being closer to the centromere, and Hfw and
Nipsnap as the distal loci. Ubc-E2H (Ubiquitin conjugating enzyme E2H) is involved in lateral inhibition and protein polyubiquitination, PhKgamma (Phosphorylase kynase enzyme) is involved in embryonic morphogenesis and glycogen metabolism and Hfw (Halfway) is involved in intracellular signal transduction and pupariation and no involvement in biological processes is yet ascribed to the Nipsnap gene although a putative role in vesicular transport has been proposed (the function of each gene is according to Flybase). Primers used for amplification and sequencing reactions are listed in Table 1. The fragments amplified correspond to partial sequences, not comprising the totality of each gene (see Table 1 for details). Distance to the nearest breakpoint $(\mathrm{Mb})$ was estimated by counting the number of cytological bands in the map of Kunze-Mühl and Müller [50] assuming that all bands contain the same DNA content $(82 \mathrm{~Kb})$ as in [51]. Distances were around $0.082 \mathrm{Mb}$ for PhKGamma and around 0.574 $\mathrm{Mb}$ for the other 3 genes (Fig. 2).

DNA amplification and sequencing was carried out as in [45] with an annealing temperature of $5 \mathrm{~s}$ at $54{ }^{\circ} \mathrm{C}$ during the amplification reaction. For each individual two sequences were obtained, from forward and reverse primers (see primer sequences in Table 1). Both sequences were assembled using SeqMan II from the Lasergene program (DNASTAR) to get one final sequence per individual. All sequences were then aligned with Clustal W, in BioEdit v.7.2 [52]. The sequence length of the total alignment for each of the four analysed genomic regions is given in Table 1 with the 5'UTR, exonic and intronic regions assigned by comparison with the orthologous sequences of Drosophila pseudoobscura retrieved from Flybase and visual inspection 
Table 1 Chromosomal location, length and primers used for each of the 4 genes studied

\begin{tabular}{|c|c|c|c|c|c|}
\hline Gene $^{a}$ & Alignment $^{\mathrm{b}}$ & Primers (Forward; Reverse) & $5^{\prime} \mathrm{UTR}$ & Exons & Introns \\
\hline PhKgamma (CG1830, GA14880) & $\begin{array}{l}831 \mathrm{bp} \\
\text { (895 bp) }\end{array}$ & $\begin{array}{l}5^{\prime}-\text { CATGACCTGGCGCAGTATTG - 3' } \\
5^{\prime} \text { - TAACAGCGGAGCGAGCAGTC - 3' }\end{array}$ & $1-240$ & $241-332$ & $333-895$ \\
\hline Ubc-E2H (CG2257, GA15327) & $\begin{array}{l}1085 \mathrm{bp} \\
(1100 \mathrm{bp})\end{array}$ & $\begin{array}{l}5^{\prime}-\text { TCACTGTAGTCGGACATGCT - 3' } \\
5^{\prime}-\text { AGGAGAGCAACGTCACAGAT - 3' }\end{array}$ & $1-662$ & $663-1100$ & - \\
\hline Hfw (CG3095, GA15922) & $\begin{array}{l}1073 \mathrm{bp} \\
(1079 \mathrm{bp})\end{array}$ & $\begin{array}{l}\text { 5' - GCATTCAAGCGGTCCGTTAA - 3' } \\
\text { 5' - TATGTTGAGGCACTTGAGCG - 3' }\end{array}$ & & 349-1079 & $1-348$ \\
\hline Nipsnap (CG9212, GA21617) & $\begin{array}{l}1095 \mathrm{bp} \\
\text { (1168 bp) }\end{array}$ & $\begin{array}{l}5^{\prime}-\text { CATGCGACTGTGAGCCTCTT - 3' } \\
5^{\prime} \text {-CGCAGCAATACAACAAGTGG - 3' }\end{array}$ & $1-411$ & $412-470 ; 1149-1168$ & $471-1148$ \\
\hline
\end{tabular}

${ }^{a}$ Gene symbol of the homologous gene in $D$. melanogaster and $D$. pseudoobscura respectively in parentheses

balignment length with D.pseudoobcura in parentheses. $5^{\prime}$ UTR, introns and exon positions are referred relative to the $D$. pseudoobscura alignment

of the resulting expected proteins. The number of males analysed per locality and inversion are presented for each gene fragment in Table 2. Our sampling strategy targeted a comparable number of males to be analysed for each population and arrangement, and as such the inversion frequencies in our samples do not match natural inversion frequencies as seen in Fig. 1.

\section{Data analysis and statistical methods}

Several standard parameters for each locality and arrangement were estimated using DnaSP v5 [53]: the number of haplotypes $(h)$, number of polymorphic sites $(S)$, the nucleotide diversity $(\pi)$, nucleotide diversity in synonymous sites and non-coding positions $\left(\pi_{\text {sil }}\right)$, heterozygosity in silent sites $\left(\Theta_{\text {sil }}\right)$ and divergence per silent site $\left(\mathrm{K}_{\mathrm{sil}}\right)$ using D. pseudoobscura as outgroup [54], with the Jukes-Cantor correction. Comparisons of the diversity values across localities and arrangements were performed with the Wilcoxon matched pairs test using Statistica v10. Genetic differentiation between or within arrangements was assessed using $F_{S T}[55]$ and $D_{x y}$ [56] as well as significant levels for Snn [57] were obtained with 10,000 replicates. False Discovery rate - FDR - correction [58] was applied to account for multiple comparisons in the differentiation analyses. Analyses were performed excluding gaps. For a visual representation of the genetic differences between arrangements and populations in the PhKgamma and $U b c-E 2 H$ gene regions, pairwise $\mathrm{F}_{\mathrm{ST}}$ values were used to compute a Principal Coordinate Analysis (pcoa in $\mathrm{R}$ package ape version 3.5 (http://ape-package.ird.fr/). A locus-by-locus analysis of Molecular variance (AMOVA) was performed on Arlequin v3.5.2 [59] to test for genetic

Table 2 Nucleotide variation for each gene and arrangement across all localities

\begin{tabular}{|c|c|c|c|c|c|c|c|c|c|c|c|c|}
\hline \multicolumn{5}{|c|}{ PhKgamma } & \multicolumn{4}{|c|}{ Ubc-E2H } & \multicolumn{2}{|l|}{ Hfw } & \multicolumn{2}{|c|}{ Nipsnap } \\
\hline & Mal & Ras & Dij & Gro & $\overline{\mathrm{Mal}}$ & Ras & Dij & Gro & $\overline{\mathrm{Mal}}$ & Gro & $\overline{\mathrm{Mal}}$ & Gro \\
\hline \multicolumn{13}{|l|}{$\overline{A_{S T}}$} \\
\hline$n$ & 9 & 9 & 10 & 10 & 9 & 10 & 10 & 8 & 7 & 9 & 9 & 10 \\
\hline$h$ & 6 & 9 & 10 & 8 & 9 & 10 & 10 & 8 & 7 & 9 & 9 & 10 \\
\hline S & 9 & 13 & 14 & 9 & 12 & 15 & 16 & 11 & 34 & 24 & 27 & 35 \\
\hline$\pi$ & 0.0031 & 0.0057 & 0.0055 & 0.0031 & 0.0038 & 0.0040 & 0.0047 & 0.0031 & 0.0105 & 0.0070 & 0.0084 & 0.0097 \\
\hline$\pi_{\mathrm{sil}}$ & 0.0034 & 0.0052 & 0.0042 & 0.0020 & 0.0038 & 0.0058 & 0.0055 & 0.0034 & 0.0142 & 0.0103 & 0.0090 & 0.0102 \\
\hline$\theta_{\text {sil }}$ & 0.0044 & 0.0055 & 0.0048 & 0.0027 & 0.0043 & 0.0071 & 0.0073 & 0.0044 & 0.0175 & 0.0120 & 0.0099 & 0.0124 \\
\hline $\mathrm{K}_{\text {sil }}$ & 0.1912 & 0.1926 & 0.1910 & 0.1887 & 0.3865 & 0.3780 & 0.3914 & 0.3799 & 0.2548 & 0.2474 & 0.4104 & 0.4138 \\
\hline \multicolumn{13}{|l|}{$\mathrm{A}_{2}$} \\
\hline$n$ & 10 & 8 & 9 & 10 & 10 & 9 & 10 & 10 & 10 & 10 & 10 & 10 \\
\hline$h$ & 5 & 3 & 3 & 5 & 8 & 9 & 8 & 6 & 10 & 10 & 9 & 10 \\
\hline s & 5 & 8 & 2 & 4 & 12 & 11 & 8 & 6 & 19 & 18 & 24 & 22 \\
\hline$\pi$ & 0.0014 & 0.0025 & 0.0008 & 0.0012 & 0.0028 & 0.0030 & 0.0020 & 0.0015 & 0.0053 & 0.0052 & 0.0068 & 0.0056 \\
\hline$\pi_{\text {sil }}$ & 0.0012 & 0.0026 & 0.0009 & 0.0008 & 0.0033 & 0.0028 & 0.0011 & 0.0014 & 0.0085 & 0.0095 & 0.0070 & 0.0057 \\
\hline$\theta_{\text {sil }}$ & 0.0021 & 0.0040 & 0.0011 & 0.0011 & 0.0050 & 0.0042 & 0.0020 & 0.0020 & 0.0102 & 0.0108 & 0.0084 & 0.0076 \\
\hline $\mathrm{K}_{\text {sil }}$ & 0.1988 & 0.1981 & 0.1999 & 0.1988 & 0.3728 & 0.3728 & 0.3714 & 0.3716 & 0.2469 & 0.2483 & 0.4144 & 0.4177 \\
\hline
\end{tabular}

Note: $n$ - sample size; $h$ - number of haplotypes; $S$ - number of polymorphic sites; $\pi$ - nucleotide diversity $\pi_{\text {sil }}$ - nucleotide diversity in silent sites (synonymous and non-coding positions); $\theta_{\text {sil }}$ - heterozygosity in silent sites $\mathrm{K}_{\text {sil }}$ - divergence per silent site using $D$. pseudoobscura as outgroup 
differentiation $\left(\mathrm{F}_{\mathrm{CT}}\right)$ between arrangements at each specific polymorphic site. A hierarchical structure was considered with two groups corresponding to the two arrangements $\left(A_{2}\right.$ vs $\left.A_{S T}\right)$, with the different localities and individuals sampled within these groups.

Gene conversion tracts (GCTs) were detected using the algorithm defined by [60]. Linkage disequilibrium (LD) between pairs of informative sites was assessed and the statistical significance analysed with a chi-square test after Bonferroni correction. These analyses were performed for each arrangement and gene region, as well as for the concatenated dataset. The overall linkage disequilibrium statistic $Z n S$ was estimated for informative sites. LD was also estimated between polymorphic informative sites through $r^{2}$ estimates. Deviations from neutrality were tested using Tajima's $D$ [61] for each chromosomal arrangement and locality. Population size changes were also determined using $R^{2}$ [62]. Statistical significance was obtained by coalescent simulations (5000 replicates) considering free recombination, as it seems most suitable when analysing $D$. subobscura genes whether located inside or outside chromosomal arrangements (see [33]). All neutrality tests were performed after removing recombinant individuals (with indication of GCTs). The DnaSP v5 program [53] was used to perform the GCTs and linkage disequilibrium analyses as well as neutrality tests.

\section{Results}

\section{Nucleotide variability and genetic differentiation}

For the two arrangements $\left(\mathrm{A}_{\mathrm{ST}}\right.$ and $\left.\mathrm{A}_{2}\right)$, we analysed the four genomic regions in the two most geographically distant localities (Málaga and Groningen), and two gene regions for the two central localities (Rasquera and Dijon) (Table 2). The two gene fragments being analysed in all localities were PhKgamma and $U b c-E 2 H$ as they showed genetic differentiation between arrangements (see Figs. 3 and 4 and Additional file 2). The genetic variation in those regions is likely to be affected by inversions to a greater extent and thus could possibly present clinal variation.

Diversity values were in general quite similar between localities for the same gene and chromosomal arrangement (see Additional file 3). Higher nucleotide diversity was found in $\mathrm{A}_{\mathrm{ST}}$ than $\mathrm{A}_{2}$ arrangements at all gene regions (Table 2 and Additional file 3), with significant differences when including information from all regions and localities for all diversity measures (Wilcoxon matched pairs test; $\mathrm{Z}=3.06, P=0.002$ for $\pi$ and $\pi_{\text {sil }} ; \mathrm{Z}=$ 2.93, $P=0.003$ for $S$; $Z=2.98, P=0.003$ for $\left.\Theta_{\text {sil }}\right)$. No differences in the divergence per silent site values $\left(K_{\text {sil }}\right)$ relative to $D$. pseudoobscura were found between the two arrangements (Wilcoxon matched pairs test; $\mathrm{Z}=0$. $24, P=0.814)$. Higher nucleotide diversity was found for
Hfw and Nipsnap (Table 2 and Additional file 3), which might possibly indicate a higher substitution rate in these two more distally located regions.

High significant genetic differentiation between the $\mathrm{A}_{\mathrm{ST}}$ and $\mathrm{A}_{2}$ arrangements was detected within each locality and also between localities for $U b c-E 2 H$ and PhKgamma (see Figs. 3 and 4 and Additional file 2) and also the concatenated dataset of all genes (see Additional file 4). On the other hand, Hfw and Nipsnap did not show significant differentiation between arrangements whether from the same or different localities (see Additional file 4). In general, no significant differentiation was observed when analysing groups of individuals carrying the same arrangement across localities with the exception of the comparison of $\mathrm{A}_{\mathrm{ST}}$ individuals from Málaga and Rasquera in PhKgamma (see Additional file 2 and Additional file 4).

As low levels of within-population variation - as is the case in PhKgamma and $U b c-E 2 H$ - can inflate $\mathrm{F}_{\mathrm{ST}}$ estimates $[63,64]$ we have used $D_{x y}$, which is not affected by such low variation. The results for each gene region were qualitatively similar to those reported above for $\mathrm{F}_{\mathrm{ST}}$, with consistently higher between-arrangement than within-arrangement differentiation for PhKgamma and $U b c-E 2 H$, although the magnitude of such differences was lower than that obtained with $\mathrm{F}_{\mathrm{ST}}$ estimates (Additional file 2). However, both statistics are highly correlated as shown by a Mantel test for PhKgamma $\left(\mathrm{r}^{2}=\right.$ $0.754, P=0.003)$ and $U b c-E 2 H(r=0.741, P=0.020)$.

Analysis of genetic differentiation, done separately in coding (exon) and non-coding (non-exon) regions for the four genes concatenated data set for Málaga and Groningen (the two geographically distant localities), showed that the significant differentiation between groups of individuals carrying different arrangements was due solely to variation in non-coding regions (see Additional file 5). Interestingly, these analyses indicated low but significant differentiation for $\mathrm{A}_{\mathrm{ST}}$ individuals from different locations in non-coding regions, but not for $\mathrm{A}_{2}$ individuals. Considering each gene region, we found high differentiation between arrangements in upstream regions for $U b c-E 2 H$ and both upstream and intronic regions for PhKgamma while no relevant differentiation was observed for the other two genes in any region analysed (Fig. 5, see also Additional file 5). Curiously, in the intronic region of PhKgamma significant genetic differentiation between $\mathrm{A}_{\mathrm{ST}}$ individuals from different localities was observed (see Additional file 5). A locus-by-locus analysis of Molecular variance (AMOVA) was performed to test the genetic differentiation $\left(\mathrm{F}_{\mathrm{CT}}\right)$ between arrangements at each specific polymorphic site using a hierarchical structure (see Methods). Again, differentiation between arrangements was observed exclusively in non-coding regions (see Additional file 6). 


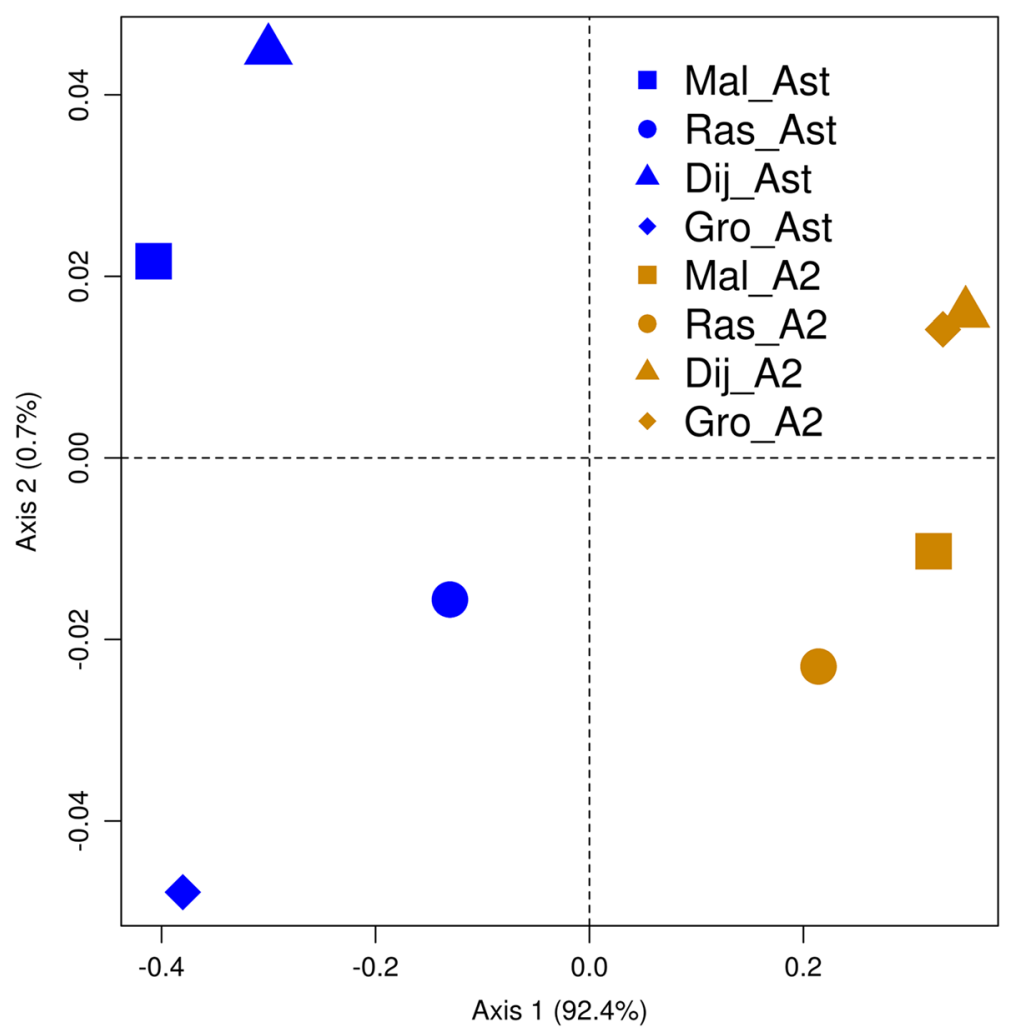

Fig. 3 Principal Coordinate analysis based on genetic differentiation $\left(F_{S T}\right)$ among arrangements and localities for the PhKgamma gene. A are represented in blue and $A_{2}$ in orange; different localities are indicated by different symbols

On average $\mathrm{F}_{\mathrm{ST}}$ values between arrangements found in the same locality or in different localities showed similar values in both PhKgamma and $U b c-E 2 H$ and the concatenated dataset (see Additional file 2 and Additional file 4; see also Figs. 3 and 4). In these regions, differentiation between arrangements was similar across localities with the exception of Rasquera for PhKgamma (see Additional file 7). However, this exception was due to the recombinant individuals from Rasquera (four individuals presenting gene conversion tracks, see below).

Pairwise genetic distances, based on the concatenated dataset of PhKgamma and $U b c-E 2 H$, were correlated with their geographic distance with Mantel tests. No significant association between genetic and geographic distances was found for individuals carrying $\mathrm{A}_{2}\left(\mathrm{r}^{2}=0.004\right.$, $P=0.462)$, or $\mathrm{A}_{\mathrm{ST}}\left(\mathrm{r}^{2}=0.017, P=0.284\right)$. Similar results are also obtained for the two genes separately (see Figs. 3 and 4). In agreement with this, no particular haplotype in any arrangement showed consistent indications of directional changes in frequency with latitude.

Gene conversion tracts (GCT) were detected only in PhKgamma and in four individuals, all of them from Rasquera. When removing individuals presenting GCTs in the PhKgamma gene, four fixed differences were detected between arrangements (position 205 in the
5'UTR, and positions 358-360 in the intron). For $U b c-E 2 H$, one fixed difference was found between the two arrangements (position 60 in the 5'UTR). No fixed differences were found in the other two genes.

\section{Linkage disequilibrium}

In general, levels of linkage disequilibrium (LD) were low in all gene regions and arrangements analysed (see Additional file 8). Using the concatenated dataset of all four genes and considering both arrangements, LD between pairs of polymorphic sites $\left(\mathrm{R}^{2}\right)$ showed only 35 significant comparisons out of a total of $2415(P<0.001$, chi-square with Bonferroni correction). Importantly, the majority of the significant LD comparisons were observed between sites from the same gene region, indicating short-range LD (see also Additional file 9). In fact, only 12 of the 35 significant comparisons corresponded to LD between distinct gene regions - 6 of them between PhKgamma and $U b c-E 2 H$ (namely between positions 358-360 bp from PhKgamma and position 60 bp from $U b c-E 2 H$, see below). In the analysis including only the individuals carrying the $A_{2}$ inversion just 3 out of a total of 496 comparisons indicated significant LD after Bonferroni correction $(P<0.001$, chi-square with Bonferroni correction). However, in these three cases, the significant LD 


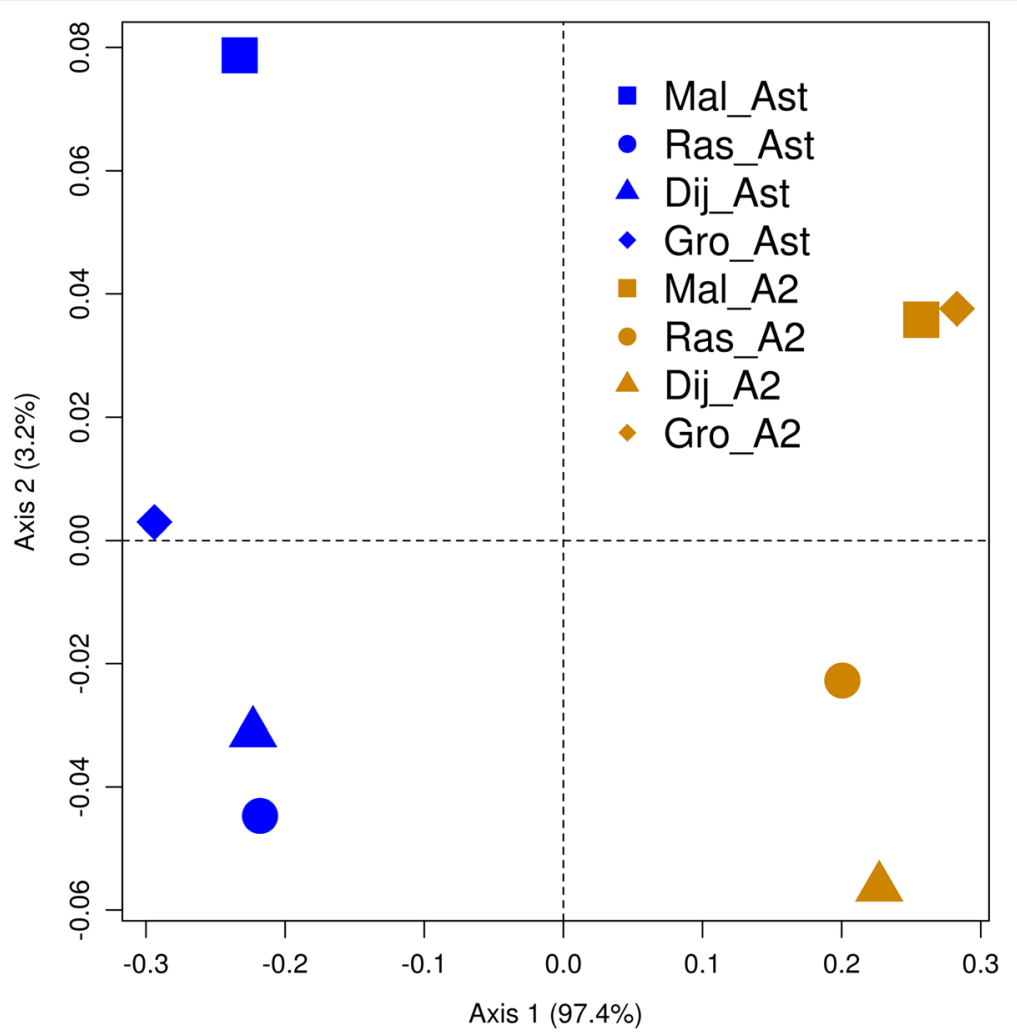

Fig. 4 Principal Coordinate analysis based on genetic differentiation $\left(F_{S T}\right)$ among arrangements and localities for the Ubc-E2H gene. $A_{S T}$ groups are represented in blue and $A_{2}$ in orange; different localities are indicated by different symbols

comparisons were always between sites within the same gene region (see Additional file 9). No significant comparisons were found between polymorphic sites for $\mathrm{A}_{\mathrm{ST}}$ individuals. Importantly, these results show that withinarrangement LD was not observed across populations.
However, fixed associations (complete LD) were found between arrangements and sites in PhKgamma (positions $205,358-360$ of the total alignment with $D$. pseudoobscura; $P<0.001$, chi-square with Bonferroni correction) and also in $U b c-E 2 H$ (position $60 ; P<0.001$, chi-square with

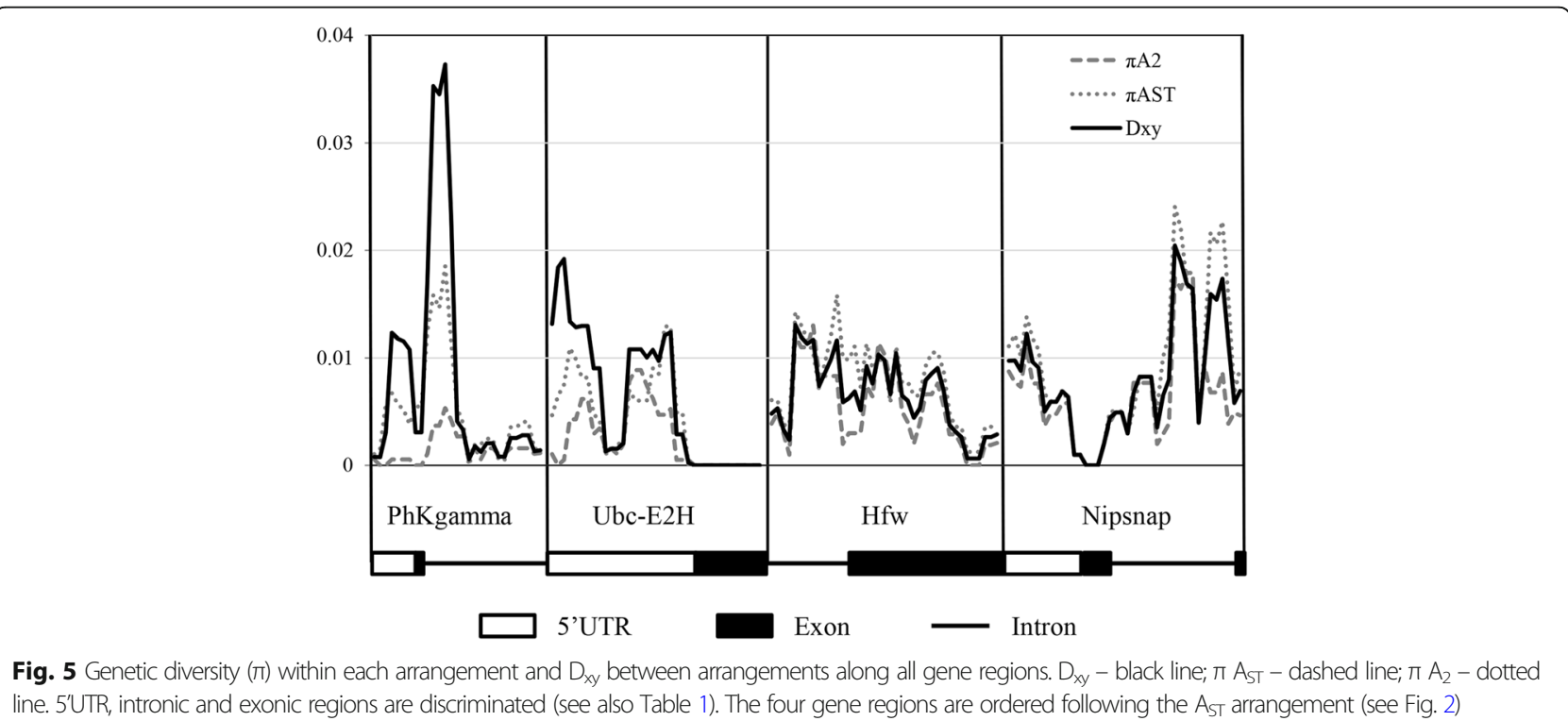


Bonferroni correction). Interestingly, all positions in PhKgamma are also in complete LD among themselves $(P$ $<0.001$, chi-square with Bonferroni correction). All these sites are located in non-coding/intronic regions. The fixed position in the $U b c-E 2 H$ gene is located 547 bp upstream from the first exon. The fixed positions at 358-360 bp in the PhKgamma gene are located in an intronic region just 26 bp downstream of the first exon. Comparison against $D$. pseudoobscura orthologous gene indicates more similarity to $\mathrm{A}_{\mathrm{ST}}$ sequences. The fixed position between arrangements at $205 \mathrm{bp}$ is within the 5'UTR region $36 \mathrm{bp}$ upstream of the start coding site of PhKgamma, and possibly within the core promoter region, despite the absence of characteristic sequence motifs such as TATA box and the TFIIB recognition element [65].

\section{Neutrality tests}

All Tajima's $D$ values were negative for each gene, arrangement and locality, and also for all locations combined (see Additional file 10). Tajima's $D$ values were generally more negative in the $A_{2}$ arrangement. $R_{2}$ values also indicated stronger deviations from neutrality in the $\mathrm{A}_{2}$ arrangement (see Additional file 10). The general scenario points to an excess of low frequency variants, consistent with a population expansion scenario, particularly in the $\mathrm{A}_{2}$ arrangement.

\section{Discussion}

$\mathrm{A}_{\mathrm{ST}}$ and $\mathrm{A}_{2}$ are respectively a cold and a warm-adapted arrangement: while $\mathrm{A}_{\mathrm{ST}}$ shows increased frequencies towards higher, colder latitudes, the opposite pattern is found for $\mathrm{A}_{2}[32,44]$, with both gene flow and local adaptation likely implicated [7]. In this study we analysed patterns of genetic variation associated with these arrangements and searched for patterns of geographical selection at the genetic level in candidate genes for thermal adaptation [40], located within or near these inverted regions.

Our study shows that these arrangements shape the patterns of genetic variation likely by reducing recombination between certain regions within chromosomes. In fact, the two proximally located regions in $\mathrm{A}_{\mathrm{ST}}$ (PhKgamma and $U b c-E 2 H$ ) presented significant differentiation between arrangements particularly in non-coding regions, regardless of the arrangement's geographical origin. Several molecular studies in Drosophila have documented significant genetic differentiation between chromosomes carrying different arrangements, particularly within inverted regions $[22,23,25,29,33,66]$ and also specifically near inversion breakpoints (e.g. $[14,21,23])$. The differentiation between arrangements that we observe could result from locally adapted alleles protected within the inversions which would be in agreement with the expectations of both the coadaptation and local adaptation hypotheses $[17,18]$. Furthermore, the fact that these differences are observed in 5'UTR and intronic regions might indicate that regulatory regions could be mostly the targets of selection (see [67]). In addition, the low differentiation between arrangements at coding regions - and the low variability observed - might be an effect of purifying selection acting in these regions. However, interpreting these patterns as being due to local adaptation is not straightforward, as neutral processes might also be involved. Non-adaptive associations between the inversion and neutral genetic variants generated during the origin and/or initial spread of the inversion could lead to longstanding differentiation and linkage disequilibrium [68], particularly near breakpoints where recombination is expectedly very low $[19,69]$. This signal is more likely to be maintained when population size is high and the inversion is young - see [19]. In our case, despite the high effective size of the species [70] the age of $\mathrm{A}_{2}$ - around 160.000 years [23], makes this expectation less likely. Moreover, the fact that microsatellite loci located in the same (proximal) regions as PhKgamma and Ubc-E2H did not show similar patterns [32] - namely the observed depletion in genetic variation and increase in differentiation between arrangements further suggests that selection might be involved in the regions analysed here.

In the specific context of the $\mathrm{A}_{\mathrm{ST}} / \mathrm{A}_{2}$ complex, Nóbrega et al. [23] also found that the extent of genetic exchange between arrangements was limited, and not sufficient to homogenize nucleotide variation in the centre of $\mathrm{A}_{2}$. In our study, we show that the reduction of recombination is not homogeneous in regions around the vicinity of the breakpoints of the $\mathrm{A}_{\mathrm{ST}} / \mathrm{A}_{2}$ complex, with significant genetic differentiation and linkage disequilibrium between inversions occurring only in the proximal region of $\mathrm{A}_{\mathrm{ST}}$. Interestingly, linkage disequilibrium occurred in one region (PhKgamma) located outside the inversion, although near the breakpoint. Other studies have suggested that suppressed recombination can extent to $2.5 \mathrm{Mb}$ or more outside inversion breakpoints [25, 71]. Functional analyses might elucidate if the non-coding variation regions found in PhKgamma and $U b c-E 2 H$ is in fact adaptive.

We did not find evidence for geographical (i.e. climatic) selection within arrangements, as very low within-arrangement genetic differentiation was detected across localities. It is possible that the differences in gene expression described previously for these genomic regions between thermal regimes [40] were controlled by other genes/sequences besides the upstream and coding regions analysed here. Importantly, the differential expression [40] was observed at the population level -between populations evolving under cold $\left(13^{\circ} \mathrm{C}\right)$ vs warm $\left(22{ }^{\circ} \mathrm{C}\right)$ regimes - and not addressed at the karyotype 
level. It is possible that the gene expression in a particular karyotype does not present clinal variation, with differential gene expression occurring between distinct karyotypes. Most interestingly, the expression of PhKgamma and Nipsnap have shown to be positively associated to latitude in semi natural populations of D. subobscura from a similar geographic gradient, but not when the same populations were maintained in a common garden [72]. Thus, although evidence is not conclusive, these gene regions could be involved in local adaptation to temperature.

Other studies in D. subobscura found very limited evidence for geographical molecular variation within inversions. Pegueroles et al. [33] also analysed candidate genes for thermal adaptation located in the $\mathrm{O}$ chromosome of $D$. subobscura and found no significant differentiation within each arrangement for individuals sampled in Barcelona (Spain) and Mount Parnes (Greece). Evidence for similar patterns of generally low withinarrangement differentiation was also described in other D. subobscura studies [73, 74] as well as studies in $D$. melanogaster ([31], but see [75]) and D. pseudoobscura [20]. However, recent evidence in D. melanogaster indicates several instances of genetic clinal variation in regions linked with inversions particularly associated with the $\operatorname{In}(3 R)$ Payne $[6,34]$, perhaps due to the higher resolution power of these later studies in terms of the genomic scan performed and number of sampling sites studied.

Within-arrangement differentiation across localities would be expected if different epistatic interactions evolved (and eventually got fixed) in different localities due to adaptation to the specific local conditions as proposed by the Dobzhansky's coadaptation model [17]. The lack of within-arrangement differentiation of the selected genes across populations suggests that population-specific epistatic interactions are not occurring in the candidate genes for thermal adaptation that we sampled. It is of course possible that other loci within the inversion might present such varying epistatic interactions in the different localities. Some studies have reported evidence of epistasis based on patterns of low LD between neighbouring sites and high LD between distant sites within the inversion $[20,31]$. Recently, indications of epistatic selection have also been obtained from observations of regions with significant genetic differentiation outside arrangements presenting strong linkage disequilibrium with the arrangements. In Atp $\alpha$ gene, another candidate gene for thermal adaptation, Pegueroles et al. [30] found strong genetic differentiation associated to the analysed arrangements of the $\mathrm{O}$ chromosome despite this gene being located outside them. Higher resolution genomic scans of adaptive variation as well as functional analyses are needed to conclusively assess the presence of relevant epistatic interactions and if these differ across populations.

\section{Conclusions}

Our genetic analysis of candidate loci for thermal adaptation [40] across geographically distinct $D$. subobscura localities of the European cline found very low withinarrangement differentiation across localities as well as no clinal patterns of genetic variation. This indicates that gene flow across the European continent has homogenized the genetic content of these potentially selected regions at a wide geographical scale. Given the strong effect of gene flow in this species, it is possible that considerable within-arrangement differentiation at a geographical scale is only maintained at relatively few loci subjected to stronger selective pressures in the different environments. In this scenario, one can argue that restricted gene flow cannot explain the observed inversion frequency clines and that local adaptation is maintaining the clinal variation in this species, counteracting the homogenizing effects of the high gene flow. We also found an important effect of the $\mathrm{A}_{\mathrm{ST}} / \mathrm{A}_{2}$ complex in shaping genetic variation and preventing relevant gene exchange in different genomic regions, although its impact was not uniform across the different regions analysed. More generally, our study adds to a body of evidence showing that inversions act as barriers to recombination even outside inversions and can potentially have a role in the spread of favourable genic combinations and consequent adaptation to local habitats.

\section{Additional files}

Additional file 1: New hybridization location for Nipsnap gene. (PDF 108 kb) Additional file 2: Measures of genetic differentiation within and between arrangements for PhKgamma and Ubc-E2H. A) Genetic differentiation (F $\left.F_{S T}\right)$ between all localities and arrangements in PhKgamma (below diagonal) and Ubc-E2H (above diagonal). B) Genetic differentiation $\left(D_{x y}\right)$ between all localities and arrangements in PhKgamma (below diagonal) and Ubc-E2H (above diagonal). (XLSX $12 \mathrm{~kb}$ )

Additional file 3: Nucleotide diversity $(\pi)$ for all genes, arrangements and localities. (PDF $178 \mathrm{~kb}$ )

Additional file 4: Genetic differentiation $\left(F_{S T}\right)$ between Malaga and Groningen in Hfw, Nipsnap and the concatenated dataset (four loci). (XLSX $10 \mathrm{~kb}$ )

Additional file 5: Genetic differentiation $\left(\mathrm{F}_{\mathrm{ST}}\right)$ for coding and non-coding regions in the concatenated dataset for all 4 genes (A) and for coding, intronic and 5' UTR regions for PhKgamma (B). (XLSX $13 \mathrm{~kb}$ )

Additional file 6: Genetic differentiation between arrangements $\left(F_{C T}\right)$ for all genes. 5'UTR, intronic and exonic regions are discriminated (see also Table 1). The four gene regions depicted are not contiguous. (PDF 238 kb)

Additional file 7: Genetic differentiation between arrangements for each locality in PhKgamma (A) and Ubc-E2H (B). (PDF 344 kb)

Additional file 8: Linkage Disequilibrium parameters for all regions and the concatenated dataset. (XLSX $10 \mathrm{~kb}$ )

Additional file 9: Linkage disequilibrium (LD) between pairs of polymorphic sites $\left(R^{2}\right)$ against the nucleotide distance between compared sites. Significant comparisons after Bonferroni correction are presented in red (see Methods for additional details). A) LD between arrangements; B) LD within $\left.A_{S T} ; C\right)$ LD within $\mathrm{A}_{2}$. (PDF $248 \mathrm{~kb}$ )

Additional file 10: Neutrality tests and test of population expansion for each arrangement and gene. (XLSX 36 kb) 


\section{Abbreviations}

AMOVA: Analysis of Molecular Variance; FDR: False discovery rate; GCT: Gene conversion track; LD: Linkage disequilibrium; PCR: Polymerase chain reaction; UTR: Untranslated region

\section{Acknowledgments}

The authors thank Margarida Matos, Inês Fragata and Josiane Santos for helpful discussions and suggestions. We also thank Gemma Calabria, João Picão-Osório and Olga Dolgova for help in inversion polymorphism scoring, Neus Perez for help in the genetic analyses and Montse Peiró for help in the in situ hybridizations.

\section{Funding}

This research was funded by projects CTM2013-48163 and CTM2017-88080 (AEI/FEDER, UE) of the Spanish Government. PS had a FCT ('Fundação para a Ciência e Tecnologia') post-doctoral grant (SFRH/BPD/36829/2007) and has currently another post-doctoral Grant from FCT (SFRH/BPD/86186/2012). MP is part of the research group 2017SGR1120 of the Generalitat de Catalunya. The funding body had no role in the design of the study and collection, analysis, and interpretation of data and in writing the manuscript.

\section{Availability of data and materials}

Data supporting the conclusions of this article are included within the article and in its additional files. All gene sequences are deposited in GenBank with accession numbers: MG877856 - MG877894 (Nipsnap), MG877895 - MG877930 (Hfw), MG877931 - MG878006 (PhKgamma) and MG878007 - MG878083 (Ubc-E2H).

\section{Authors' contributions}

PS participated in the experiment design, sample and data collection, data analysis and writing of the manuscript. MP participated in the experiment design, sample collection and revised the manuscript. Both authors have read and approved the final version of the manuscript.

\section{Ethics approval and consent to participate}

Not applicable.

\section{Competing interests}

The authors declare that they have no competing interests.

\section{Publisher's Note}

Springer Nature remains neutral with regard to jurisdictional claims in published maps and institutional affiliations.

\section{Received: 26 July 2017 Accepted: 11 April 2018}

\section{Published online: 24 April 2018}

\section{References}

1. Hoffmann AA, Rieseberg LH. Revisiting the impact of inversions in evolution: from population genetic markers to drivers of adaptive shifts and speciation? Annu Rev Ecol Evol Syst. 2008;39:21-42.

2. Kirkpatrick M. How and why chromosome inversions evolve. PLoS Biol. 2010;8(9):e1000501.

3. Prevosti A, Ribo G, Serra L, Aguade M, Balanyà J, Monclus M, et al. Colonization of America by Drosophila subobscura: experiment in natural populations that supports the adaptive role of chromosomal-inversion polymorphism. Proc Natl Acad Sci U S A. 1988:85:5597-600.

4. Umina PA, Weeks AR, Kearney MR, McKechnie SW, Hoffmann AA. A rapid shift in a classic clinal pattern in Drosophila reflecting climate change. Science. 2005;308:691-3.

5. Balanyà J, Oller JM, Huey RB, Gilchrist GW, Serra L. Global genetic change tracks global climate warming in Drosophila subobscura. Science. 2006;313: 1773-5.

6. Kapun M, Fabian DK, Goudet J, Flatt T, Flatt T. Genomic evidence for adaptive inversion clines in Drosophila melanogaster. Mol Biol Evol. 2016:33:1317-36.

7. Rezende E, Balanyà J, Rodríguez-Trelles F, Rego C, Fragata I, Matos M, et al. Climate change and chromosomal inversions in Drosophila subobscura. Clim Res. 2010:43:103-14.

8. Ayala D, Ullastres A, Gonzalez J. Adaptation through chromosomal inversions in Anopheles. Front Genet. 2014:5:129.

9. Lowry DB, Willis JH. A widespread chromosomal inversion polymorphism contributes to a major life-history transition, local adaptation, and reproductive isolation. PLoS Biol. 2010;8(9):e1000500.
10. Simões P, Fragata I, Lopes-Cunha M, Lima M, Kellen B, Bárbaro M, et al. Wing trait-inversion associations in Drosophila subobscura can be generalized within continents, but may change through time. J Evol Biol. 2015;28:2163-74.

11. Hoffmann AA, Weeks AR. Climatic selection on genes and traits after a 100 year-old invasion: a critical look at the temperate-tropical clines in Drosophila melanogaster from eastern Australia. Genetica. 2007;129:133-47.

12. Kennington WJ, Hoffmann AA, Partridge L. Mapping regions within cosmopolitan inversion In(3R)Payne associated with natural variation in body size in Drosophila melanogaster. Genetics. 2007;177:549-56.

13. Rako L, Anderson AR, Sgrò CM, Stocker AJ, Hoffmann AA. The association between inversion In(3R)Payne and clinally varying traits in Drosophila melanogaster. Genetica. 2006;128:373-84.

14. Kapun M, Van Schalkwyk H, McAllister B, Flatt T, Schlötterer C. Inference of chromosomal inversion dynamics from pool-Seq data in natural and laboratory populations of Drosophila melanogaster. Mol Ecol. 2014;23:1813-27.

15. Fragata I, Lopes-Cunha M, Bárbaro M, Kellen B, Lima M, Santos MA, et al. How much can history constrain adaptive evolution? A real-time evolutionary approach of inversion polymorphisms in Drosophila subobscura. J Evol Biol. 2014;27:2727-38

16. Santos J, Pascual M, Fragata I, Simões P, Santos MA, Lima M, et al. Tracking changes in chromosomal arrangements and their genetic content during adaptation. J Evol Biol. 2016;29:1151-67.

17. Dobzhansky T. Genetics of natural populations. XIX. Origin of heterosis through natural selection in populations of Drosophila pseudoobscura. Genetics. 1950;35:288-302.

18. Kirkpatrick M, Barton N. Chromosome inversions, local adaptation and speciation. Genetics. 2006;173:419-34.

19. Guerrero RF, Rousset F, Kirkpatrick M. Coalescent patterns for chromosomal inversions in divergent populations. Phil Trans R Soc B. 2012;367:430-8.

20. Schaeffer SW, Goetting-Minesky MP, Kovacevic M, Peoples JR, Graybill JL, Miller JM, et al. Evolutionary genomics of inversions in Drosophila pseudoobscura: evidence for epistasis. Proc Natl Acad Sci U S A. 2003;100:8319-24.

21. Laayouni $H_{\text {, Hasson }} \mathrm{E}$, Santos M, Fontdevila $A$. The evolutionary history of Drosophila buzzatii. XXXV. Inversion polymorphism and nucleotide variability in different regions of the second chromosome. Mol Biol Evol. 2003:20:931-44.

22. Schaeffer SW, Anderson WW. Mechanisms of genetic exchange within the chromosomal inversions of Drosophila pseudoobscura. Genetics. 2005;171:1729-39.

23. Nóbrega C, Khadem M, Aguadé M, Segarra C. Genetic exchange versus genetic differentiation in a medium-sized inversion of Drosophila: the $A_{2} /$ $A_{S T}$ arrangements of Drosophila subobscura. Mol Biol Evol. 2008;25:1534-43.

24. Corbett-Detig RB, Hartl DL. Population genomics of inversion polymorphisms in Drosophila melanogaster. PLoS Genet. 2012;8(12):e1003056.

25. Mcgaugh SE, Noor MAF. Genomic impacts of chromosomal inversions in parapatric Drosophila species. Phil Trans R Soc B. 2012:367:422-9.

26. Cheng C, White BJ, Kamdem C, Mockaitis K, Costantini C, Hahn MW, et al. Ecological genomics of Anopheles gambiae along a latitudinal Cline: a population-resequencing approach. Genetics. 2012;190:1417-32.

27. Stump AD, Pombi M, Goeddel L, Ribeiro JMC, Wilder JA, Torre AD, et al. Genetic exchange in 2La inversion heterokaryotypes of Anopheles gambiae. Insect Mol Biol. 2007:16:703-9.

28. White BJ, Cheng C, Sangaré D, Lobo NF, Collins FH, Besansky NJ. The population genomics of trans-specific inversion polymorphisms in Anopheles gambiae. Genetics. 2009;183:275-88

29. Munté A, Rozas J, Aguadé M, Segarra C. Chromosomal inversion polymorphism leads to extensive genetic structure: a multilocus survey in Drosophila subobscura. Genetics. 2005:169:1573-81.

30. Pegueroles C, Ferrés-Coy A, Marti-Solano M, Aquadro CF, Pascual M, Mestres F. Inversions and adaptation to the plant toxin ouabain shape DNA sequence variation within and between chromosomal inversions of Drosophila subobscura. Sci Rep. 2016;6:23754

31. Kennington WJ, Partridge L, Hoffmann AA. Patterns of diversity and linkage disequilibrium within the cosmopolitan inversion In(3R)Payne in Drosophila melanogaster are indicative of coadaptation. Genetics. 2006;172:1655-63.

32. Simões P, Calabria G, Picão-Osório J, Balanyà J, Pascual M. The genetic content of chromosomal inversions across a wide latitudinal gradient. PLoS One. 2012; 7:e51625

33. Pegueroles C, Aquadro CF, Mestres F, Pascual M. Gene flow and gene flux shape evolutionary patterns of variation in Drosophila subobscura. Heredity. 2013:110:520-9.

34. Rane RV, Rako L, Kapun M, Lee SF, Hoffmann AA. Genomic evidence for role of inversion 3RP of Drosophila melanogaster in facilitating climate change adaptation. Mol Ecol. 2015;24:2423-32. 
35. Rodríguez-Trelles F, Tarrío R, Santos M. Genome-wide evolutionary response to a heat wave in Drosophila. Biol Lett. 2013;9:20130228.

36. Rego C, Balanya J, Fragata I, Matos M, Rezende E, Santos M. Clinal patterns of chromosomal inversion polymorphisms in Drosophila subobscura are partly associated with thermal preferences and heat stress resistance. Evolution. 2010;64:385-97.

37. Dolgova O, Rego C, Calabria G, Balanyà J, Pascual M, Rezende E, et al. Genetic constraints for thermal coadaptation in Drosophila subobscura. BMC Evol Biol. 2010;10:363.

38. Calabria G, Dolgova O, Rego C, Castañeda LE, Rezende EL, Balanyà J, et al. Hsp70 protein levels and thermotolerance in Drosophila subobscura: a reassessment of the thermal co-adaptation hypothesis. J Evol Biol. 2012; 25:691-700.

39. Fragata I, Balanyà J, Rego C, Matos M, Rezende EL, Santos M. Contrasting patterns of phenotypic variation linked to chromosomal inversions in native and colonizing populations of Drosophila subobscura. J Evol Biol. 2010;23: $112-23$.

40. Laayouni H, García-Franco F, Chávez-Sandoval BE, Trotta V, Beltran S, Corominas $M$, et al. Thermal evolution of gene expression profiles in Drosophila subobscura. BMC Evol Biol. 2007:7:42

41. Fuller ZL, Haynes GD, Richards S, Schaeffer SW. Genomics of natural populations: how differentially expressed genes shape the evolution of chromosomal inversions in Drosophila pseudoobscura. Genetics. 2016; 204:287-301.

42. Pascual M, Aquadro CF, Soto V, Serra L. Microsatellite variation in colonizing and palearctic populations of Drosophila subobscura. Mol Biol Evol. 2001;18: 731-40.

43. Pascual M, Chapuis MP, Mestres F, Balanyà J, Huey RB, Gilchrist GW, et al Introduction history of Drosophila subobscura in the new world: a microsatellite-based survey using ABC methods. Mol Ecol. 2007;16:3069-83.

44. Balanyà J, Serra L, Gilchrist GW, Huey RB, Pascual M, Mestres F, et al. Evolutionary pace of chromosomal polymorphism in colonizing populations of Drosophila subobscura: an evolutionary time series. Evolution. 2003;57: 1837-45.

45. Calabria G, Máca J, Bachli G, Serra L, Pascual M. First records of the potential pest species Drosophila suzukii (Diptera: Drosophilidae) in Europe. J Appl Entomol. 2012;136:139-47.

46. Huey RB, Pascual M. Partial thermoregulatory compensation by a rapidly evolving invasive species along a latitudinal cline. Ecology. 2009;90:1715-20.

47. Krimbas C. The inversion polymorphism of Drosophila subobscura. In: Krimbas C, Powell J, editors. Drosophila inversion polymorphism. Boca Raton (FL): CRC press; 1992. p. 127-220

48. Pascual M, Balanyà J, Latorre A, Serra L. Analysis of the variability of Drosophila azteca and D. athabasca populations revealed by randomly amplified polymorphic DNA. J Zool Syst Evol Res. 1997;35:159-64.

49. Laayouni H, Santos M, Fontdevila A. Toward a physical map of Drosophila buzzatii: use of randomly amplified polymorphic DNA polymorphisms and sequence tagged site landmarks. Genetics. 2000;156:1797-816.

50. Kunze-Mühl E, Müller E. Weitere Untersuchungen über die chromosomale Struktur und die naturlichen Strukturtypen von Drosophila subobscura coll. Chromosoma. 1957;9:559-70.

51. Pegueroles C, Araúz PA, Pascual M, Mestres F. A recombination survey using microsatellites: the $\mathrm{O}$ chromosome of Drosophila subobscura. Genetica. 2010;138:795-804

52. Hall TA. BioEdit: a user-friendly biological sequence alignment editor and analysis program for windows 95/98/NT. Nucleic Acids Symp. 1999;41:95-8.

53. Librado P, Rozas J. DnaSP v5: a software for comprehensive analysis of DNA polymorphism data. Bioinformatics. 2009;25:1451-2.

54. Nei M, Gojobori T. Simple methods for estimating the numbers of synonymous and nonsynonymous nucleotide substitutions. Mol Biol Evol. 1986;3:418-26.

55. Hudson R, Slatkin M, Maddison W. Estimation of levels of gene flow from DNAsequence data. Genetics. 1992;132:583-9.

56. Nei M. Molecular evolutionary genetics. New York: Columbia University Press; 1987

57. Hudson R. A new statistic for detecting genetic differentiation. Genetics. 2000;155:2011-4

58. Benjamini $Y$, Yekutieli $D$. The control of the false discovery rate in multiple testing under dependency. Ann Stat. 2001;29:1165-88.

59. Excoffier $L$, Lischer $H$. Arlequin suite ver 3.5: a new series of programs to perform population genetics analyses under Linux and windows. Mol Ecol Resour. 2010;10:564-7.
60. Betrán E, Rozas J, Navarro A, Barbadilla A. The estimation of the number and the length distribution of gene conversion tracts from population DNA sequence data. Genetics. 1997;146:89-99.

61. Tajima F. Statistical method for testing the neutral mutation hypothesis by DNA polymorphism. Genetics. 1989;123:585-95.

62. Ramos-Onsins SE, Rozas J. Statistical properties of new neutrality tests against population growth. Mol Biol Evol. 2002;19:2092-100.

63. Charlesworth B. Measures of divergence between populations and the effect of forces that reduce variability. Mol Biol Evol. 1998;15:538-43.

64. Cruickshank TE, Hahn MW. Reanalysis suggests that genomic islands of speciation are due to reduced diversity, not reduced gene flow. Mol Ecol. 2014;23:3133-57.

65. Butler JEF, Kadonaga JT. The RNA polymerase II core promoter: a key component in the regulation of gene expression. Genes Dev. 2002;16:2583-92.

66. Corbett-Detig RB, Cardeno C, Langley CH. Sequence-based detection and breakpoint assembly of polymorphic inversions. Genetics. 2012;192:131-7.

67. Palsson A, Wesolowska N, Reynisdóttir S, Ludwig MZ, Kreitman M. Naturally occurring deletions of hunchback binding sites in the even-skipped stripe 3+7 enhancer. PLoS One. 2014;9:e91924.

68. Ishii K, Charlesworth B. Associations between allozyme loci and gene arrangements due to hitch-hiking effects of new inversions. Genet Res. 1977:30:93-106.

69. Navarro A, Barbadilla A, Ruiz A. Effect of inversion polymorphism on the neutral nucleotide variability of linked chromosomal regions in Drosophila. Genetics. 2000;155:685-98.

70. Pascual M, Schug MD, Aquadro CF. High density of long dinucleotide microsatellites in Drosophila subobscura. Mol Biol Evol. 2000;17:1259-67. https://doi.org/10.1093/oxfordjournals.molbev.a026409.

71. Pegueroles C, Ordóñez V, Mestres F, Pascual M. Recombination and selection in the maintenance of the adaptive value of inversions. J Evol Biol. 2010;23: 2709-17.

72. Porcelli D, Westram AM, Pascual M, Gaston KJ, Butlin RK, Snook RR. Gene expression clines reveal local adaptation and associated trade-offs at a continental scale. Sci Rep. 2016;6:32975.

73. Rozas J, Segarra C, Zapata C, Alvarez G, Aguadé M. Nucleotide polymorphism at the rp49 region of Drosophila subobscura: lack of geographic subdivision within chromosomal arrangements in Europe. J Evol Biol. 1995:367:355-67.

74. Sánchez-Gracia A, Rozas J. Molecular population genetics of the OBP83 genomic region in Drosophila subobscura and D. guanche: contrasting the effects of natural selection and gene arrangement expansion in the patterns of nucleotide variation. Heredity. 2011;106:191-201.

75. Kennington WJ, Hoffmann AA. Patterns of genetic variation across inversions: geographic variation in the $\ln (2 \mathrm{~L}) \mathrm{t}$ inversion in populations of Drosophila melanogaster from eastern Australia. BMC Evol Biol. 2013;13:100.

\section{Ready to submit your research? Choose BMC and benefit from:}

- fast, convenient online submission

- thorough peer review by experienced researchers in your field

- rapid publication on acceptance

- support for research data, including large and complex data types

- gold Open Access which fosters wider collaboration and increased citations

- maximum visibility for your research: over $100 \mathrm{M}$ website views per year

At BMC, research is always in progress.

Learn more biomedcentral.com/submissions 